Pacific Journal of Mathematic 


\title{
DIAGONAL SUBMATRICES OF MATRIX MAPS
}

\author{
AlFred E. TONG
}

The first question answered in this paper is: if $A: \lambda \rightarrow \mu$ is a linear operator between sequence spaces, with a matrix representation $\left(a_{\imath j}\right)$, does it follow that the associated diagonal matrix $\left(\alpha_{i j} \delta_{\jmath \jmath}\right)$ maps $\lambda$ into $\mu$ ? An affirmative answer is given if $\lambda$ is a normal (or monotone) sequence space and $\mu$ is a perfect sequence space.

Morever, if $\lambda, \mu$ are normed sequence spaces, under what conditions will the following inequality hold for all matrix maps $\left(a_{i \jmath}\right)$ from $\lambda$ to $\mu:\left\|\left(a_{\imath \jmath}\right)\right\| \geqq\left\|\left(a_{\imath \jmath} \delta_{\imath j}\right)\right\|$ (where $\|\cdot\|$ denotes the operator sup norm)?

We apply our answer to the first problem to give another proof for a theorem of S. Mazur.

Section 1 gives the definitions, notations, and some computations. Section 2 considers the first question. Section 3 gives the application to Mazur's theorem. Definitions of terms relating to sequence spaces are from Köthe (1).

1. Vector spaces of sequences, over the real or complex number system, which contain all finitely supported sequences are called sequence spaces and are denoted by $\lambda$ and $\mu$. Let $x$ be a sequence $(x(1), \cdots$, $x(i), \cdots)$ in $\lambda$. By the support of $x$ we mean the set of all indices $i$ for which $x(i) \neq 0$. We use $m$ to denote the space of all bounded sequences and $\omega$ to denote the space of all sequences. We say that a sequence of vectors $x_{1}, \cdots, x_{k}, \cdots$, in $\lambda$ is disjointly supported if the family of supports $S(k)$ of $x_{k}$ is disjoint and we use $x_{1} \vee x_{2} \vee \ldots$ $\vee x_{k} \vee$ to denote the sequence $x \in \omega$ defined by $x(i)=x_{l k}(i)$ if there is a $k$ for which $i \in S(k)$ and $x(i)=0$, otherwise.

Let $c$ denote a sequence $(c(1), \cdots, c(i), \cdots)$ in $\omega$. The sequence $(c(1) x(1), \cdots, c(i) x(i), \cdots)$ is denoted by $c \cdot x$. A sequence space $\lambda$ which satisfies: $c \cdot x \in \lambda$ for all $c \in m$ and all $x \in \lambda$ is called a normal sequence space.

A monotone sequence space $\lambda$ is a sequence space satisfying $c \cdot x \in \lambda$ for all $x \in \lambda$ and $c \in m$ where $c(i)=1,-1$, or 0 (all $i$ ). The sequence $(1,1, \cdots, 1, \cdots)$ is denoted by $\overline{1}$.

$\delta_{i j}$ denotes the Kronecker delta. If $S$ is a set of indices and $\lambda$ is a normal sequence space, the linear projection $\pi_{s}: \lambda \rightarrow \lambda$ is defined by setting $\pi_{s}(x)$ to be the vector in $\lambda$ whose $i$-th coordinate is $x_{i}$ when $i \in S$ and is 0 when $i \in S$. If $S$ is the set of all indices $m \leqq i \leqq n$, we shall write $\pi_{[m, n]}$.

DeFinition 1.1. Let $\lambda, \mu$ be sequence spaces. A linear map 
$A: \lambda \rightarrow \mu$ is said to have matrix representation $\left(a_{i j}\right)$ if $\sum_{j} a_{i j} x(j)$ converges for all $x \in \lambda$ and if

$$
A(x)=\left(\sum_{j} a_{1 j} x(j), \cdots, \sum_{j} a_{i j} x(j), \cdots\right) .
$$

By the associated diagonal of $A$, we mean the linear map $D: \lambda \rightarrow \omega$ with matrix representation $\left(\delta_{i j} a_{i j}\right)$. We write $r_{i}$ to denote the sequence consisting of the $i$-th row of $\left(a_{i j}\right)$ and $c_{j}$ to denote the sequence consisting of the $j$-th column of $\left(a_{i j}\right)$.

Linear operators with matrix representation are referred to as matrix maps.

A sign distribution $\sigma$ on $n$ places is any sequence $\sigma=\left(x_{1}, \cdots\right.$, $x_{i}, \cdots, x_{n}$ ) where $x_{i}= \pm 1$ for all $i$. Two sign distributions $\sigma, \tau$ are said to be distinct if $\sigma \neq \tau$ and $\sigma \neq-\tau$.

Lemma 1.2. Let $\mathscr{S}=\left\{\sigma_{1}, \cdots, \sigma_{i}, \cdots, \sigma_{2^{n-1}}\right\}$ be a family of $2^{n-1}$ distinct sign distributions on $n$ places. Then:

$$
\sum_{1 \leqq i \leqq 2^{n-1}}\left|\sum_{1 \leqq k \leqq n} \sigma_{i}(k) x(k)\right| \geqq 2^{n-1} \max \{|x(1)|, \cdots,|x(n)|\},
$$

where $x(1), \cdots, x(n)$ are complex numbers.

For the proof, see (1.3) of [3] where one uses the triangle inequality instead of the Lemma (1.2) in [3].

Lemma 1.3. Let $\lambda, \mu$ be sequence spaces of dimension $n$. Let $A: \lambda \rightarrow \mu$ be a matrix map represented by $\left(a_{i j}\right)$. For each $u \in \lambda, v^{\prime} \in \mu^{x}$, we can find a sign distribution $\sigma$ on $n$ places and $y^{\prime} \in \mu^{x}$ so that:

(a) $\left|y^{\prime}(l)\right|=\left|v^{\prime}(l)\right|$ for all $l=1,2, \cdots, n$.

(b) If $x$ is defined by $x(k)=\sigma(k) u(k)$, then

$$
\left(A(x), y^{\prime}\right) \geqq \sum_{1 \leqq k \leqq n}\left|a_{k k} u(k) v^{\prime}(k)\right| \text {. }
$$

Proof. Let $\mathscr{S}=\left\{\sigma_{1}, \cdots, \sigma_{2^{n-1}}\right\}$ be a family of $2^{n-1}$ distinct sign distributions on $n$ places. Lemma (1.2) gives:

$$
\left|v^{\prime}(l)\right| \sum_{1 \leqq i 2^{n-1}}\left|\sum_{1 \leqq k \leqq n} \sigma_{i}(k) a_{l k} u(k)\right| \geqq 2^{n-1}\left|a_{l l} u(l)\right| \cdot\left|v^{\prime}(l)\right|
$$

for all $l=1, \cdots, n$. Hence, we can find a sign distribution $\sigma_{1}$ so that:

$$
\sum_{1 \leqq l \leqq n}\left|v^{\prime}(l)\right|\left|\sum_{1 \leqq k \leqq n} \sigma_{i}(k) a_{l k} u(k)\right| \geqq \sum_{1 \leqq l \leqq n}\left|a_{l l} u(l)\right| \cdot\left|v^{\prime}(l)\right| .
$$

Choose $y^{\prime}$ to satisfy: 


$$
y^{\prime}(l) \sum_{1 \leqq k \leqq n} \sigma_{i}(k) a_{l k} u(k)=\left|v^{\prime}(l)\right|\left|\sum_{1 \leqq k \leqq n} \sigma_{i}(k) a_{l k} u(k)\right| .
$$

Thus, $\left|y^{\prime}(l)\right|=\left|v^{\prime}(l)\right|$. By (1.3.1), we get:

$$
\left(A(x), y^{\prime}\right) \geqq \sum_{1 \leqq l \leqq n}\left|a_{l l} u(l) v^{\prime}(l)\right| \text {. }
$$

Definition 1.4. A normed (Banach) sequence space $\lambda$ is said to be a normed (Banach) ideal if it is a normal sequence space satisfying:

$$
\|c \cdot x\| \leqq\|x\| \sup \{|c(i)|: i=1,2, \cdots\}
$$

for all $c \in m, x \in \lambda$. A normed monotone sequence space is said to be monotonely normed if (1.4.1) holds for all $c \in m$ satisfying $c(i)=1,-1$, or 0 (all $i$ ).

REMARK 1.5. If $\lambda$ is a monotonely normed sequence space of dimension $n$ and $\mu$ is a normed ideal of dimension $n$, then Lemma (1.3) shows that: $\|A\| \geqq\|D\|$ where $D$ is the associated diagonal of $A$.

Definition 1.6. Let $A=\left(a_{i j}\right)$ be a matrix. Let

$$
1=m(1) \leqq n(1)<\cdots<m(k) \leqq n(k)<\cdots
$$

be a sequence of indices. The submatrix of $A$ whose $(i, j)$-th entry is $a_{i j}$ when there is a $k$ for which $m(k) \leqq i, j \leqq n(k)$ and whose $(i, j)$-th entry is 0 , otherwise, is called the diagonal block submatrix of $A$ determined by $m(k), n(k)$, and is denoted by $B$. Denote

$$
\pi_{[m(k), n(k)]} A \pi_{[m(k), n(k)]}
$$

by $A^{[k]}$. Note that $A^{[k]}=B^{[k]}$.

2. Throughout this section, $\lambda$ and $\mu$ are spaces of sequences over the real numbers.

Theorem 2.1. Let $\lambda$ be a monotone sequence space. Let $\mu$ be any sequence space. If $A: \lambda \rightarrow \mu$ is a matrix map and $D$ is the associated diagonal submatrix, then $D(\lambda) \subset \mu^{x x}$.

Proof. Suppose $D(\lambda) \not \subset \mu^{x x}$. Then we can find $u \in \lambda, v^{\prime} \in \mu^{x}$ so that

$$
\sum_{i}\left|a_{i i} u(i) v^{\prime}(i)\right|=\infty \text {. }
$$

Define a sequence of indices

$$
1=m(1) \leqq n(1)<\cdots<m(k) \leqq n(k) \cdots
$$


inductively. Suppose $\left(a_{i j}\right)$ is the matrix representation of $A$. Set $m(1)=1$. Assume: $m(1) \leqq \cdots<m(k)$ have been chosen.

(2.1.1) Choose $n(k)$ so that

(1) $m(k) \leqq n(k)$

(2) $\sum_{m(k) \leqq i \leqq n(k)}\left|a_{i i} u(i) v^{\prime}(i)\right|>k$.

(2.1.2) Choose $m(k+1)$ to satisfy

(1) $n(k)<m(k+1)$

(2) $\sum_{m(k+1) \leqq j<\infty}\left|a_{i j} u(j) v^{\prime}(i)\right|<1 / 2^{k} n(k)$ whenever $m(k) \leqq i \leqq n(k)$

(3) $\sum_{m(k+1) \leqq i<\infty}\left|a_{i j} u(j) v^{\prime}(i)\right|<1 / 2^{k} n(k)$ whenever $m(k) \leqq j \leqq n(k)$. Here, we used the fact that the $i$-th row $r_{i}$ of $\left(a_{i j}\right)$ is a sequence in $\lambda^{x}$ to obtain (2) and the assumption that $A(\lambda) \subset \mu$ and that $v^{\prime} \in \mu^{x}$ to obtain (3).

Let $\sigma_{1}, \cdots, \sigma_{k}, \cdots$, be elements in $m$ so that $\sigma_{k}$ is supported on [m(k), $n(k)],\left|\sigma_{k}(j)\right|=1$ if $j$ is in the support, and so that

$$
\sum_{m(k) \leqq i \leqq n(k)}\left|v^{\prime}(i)\right|\left|\sum_{m(k) \leqq j \leqq n(k)} a_{i j} \sigma_{k}(j) u(j)\right| \geqq \sum_{m(k) \leqq i \leqq n(k)}\left|a_{i i} u(i) v^{\prime}(i)\right| .
$$

Here, we used (1.3.1). Hence, by (2.1.1),

$$
\begin{gathered}
\sum_{k}\left(\sum_{m(k) \leqq i \leqq n(k)}\left|v^{\prime}(i)\right|\left|\sum_{m(k) \leqq j \leqq n(k)} a_{i j} \sigma_{k}(j) u(j)\right|\right) \\
\geqq \sum_{k} \sum_{m(k) \leqq i \leqq n(k)}\left|a_{i i} u(i) v^{\prime}(i)\right|=\infty .
\end{gathered}
$$

If we can show that the term on the left, which we denote by $\Delta$, is finite, then a contradiction results and the hypothesis $D(\lambda) \not \subset \mu^{x x}$ is false.

Define $\sigma=\sigma_{1} \vee \cdots \vee \sigma_{k} \vee \cdots$. Let $\tau \in m$ be defined by

$$
\tau(i)=\operatorname{sign}\left(v(i) \sum_{m(k) \leqq j \leqq n(k)} a_{i j} \sigma_{k}(j) u(j)\right)
$$

whenever $m(k) \leqq i \leqq n(k)$ and by $\tau(i)=0$, otherwise. Let

$$
S_{k}=\left\{i: m\left(k^{\prime}\right) \leqq i \leqq n\left(k^{\prime}\right) \text { for some } k^{\prime}>k\right\} \text {. }
$$

Observe that (2.1.2) (2) and (3) give:

$$
\begin{aligned}
& \sum_{k} \sum_{j \in S_{k}} \sum_{m(k) \leqq i \leqq n(k)}\left|a_{i j} u(j) v^{\prime}(i)\right|<\infty \\
& \sum_{k} \sum_{i \in S_{k}} \sum_{m(k) \leqq j \leqq n(k)}\left|a_{i j} u(j) v^{\prime}(i)\right|<\infty
\end{aligned}
$$

By inspection, $\Delta=\left(A(\sigma \cdot u), \tau \cdot v^{\prime}\right)$

$$
\begin{aligned}
& -\sum_{k} \sum_{j \in S_{k}} \sum_{m(k) \leqq i \leqq n(k)} a_{i j} u(j) v^{\prime}(i) \\
& -\sum_{k} \sum_{i \in S_{k}} \sum_{m(k) \leqq j \leqq n(k)} a_{i j} u(j) v^{\prime}(i) .
\end{aligned}
$$


Since $\lambda$ and $\mu^{x}$ are monotone sequence spaces and since $A(\lambda) \subset \mu$, we must have: $\sigma \cdot u \in \lambda$ so that

$$
\left(A(\sigma \cdot u), \tau \cdot v^{\prime}\right)<\infty .
$$

Thus $\Delta<\infty$.

Lemma 2.2. Let $X$ be a normed space. Let $\left(a_{i j}\right)$ be an $n \times n$ matrix of vectors in $X$. Then there is a sign distribution $\sigma$ on $n$ places and a vector $y^{\prime} \in l_{\infty}^{n}$ where $\left|y^{\prime}(i)\right|=1$ for $1 \leqq i \leqq n$ such that:

$$
\left\|\sum_{1<i \leqq n} y(i) \sum_{1 \leqq j \leqq n} \sigma(j) a_{i j}\right\| \geqq\left\|\sum_{1 \leqq k \leqq n} a_{k k}\right\| \text {. }
$$

Proof. For each $x^{\prime} \in X^{\prime}$, define a matrix $A$ of real numbers by setting the $i, j$-th term to be $\left(a_{i j}, x^{\prime}\right)$. Choose $u=\overline{1}, v^{\prime}=\overline{1}$ in (1.3) to get the existence of a sign distribution $\sigma$ on $n$ places and a sequence $y^{\prime}$ so that $\left|y^{\prime}(i)\right|=1$ for all $i=1,2, \cdots, n$ and

$$
\begin{aligned}
\left(\sum_{i} y^{\prime}(i) \sum_{j} \sigma(j) a_{i j}, x^{\prime}\right) & =\left(A(\sigma), y^{\prime}\right) \\
& \geqq \sum_{1 \leqslant k \leqslant n}\left|\left(a_{k k}, x^{\prime}\right)\right| .
\end{aligned}
$$

By the Hahn-Banach theorem, this implies:

$$
\left\|\sum_{i} y^{\prime}(i) \sum_{j} \sigma(j) a_{i j}\right\| \geqq\left\|\sum_{1 \leqq k \leqq n} a_{k k}\right\| .
$$

Theorem 2.3. Let $1=m(1) \leqq n(1)<\cdots m(k) \leqq n(k) \cdots$ be a sequence of indices. Let $\lambda$ be a monotonely normed sequence space and $\mu$ be a sequence space. Let $A: \lambda \rightarrow \ell$ be a matrix map. Then, the associated diagonal block submatrix $B$, determined by $\{m(k), n(k)\}$, of $A$ satisfies: $B(\lambda) \subset \mu^{x x}$ and $\|A\| \geqq\|B\|$ where $\|\cdot\|$ denotes the operator sup norm.

Proof. Let $\pi_{k}^{1}$ denote $\pi_{[m(k), n(k)]}$ and $\pi_{k}^{2}$ denote $\pi_{[n(k)+1, m(k+1)-1]}$. Define $a_{i j}$ to be $\pi_{l}^{1} A \pi_{k}^{1}, \pi_{l}^{2} A \pi_{l}^{1}, \pi_{l}^{1} A \pi_{k}^{2}$, or $\pi_{l}^{2} A \pi_{k}^{2}$ depending on whether

(1) $i=2 k-1$ and $j=2 l-1$,

(2) $i=2 k-1$ and $j=2 l$,

(3) $i=2 k$ and $j=2 l-1$ or

(4) $i=2 k$ and $j=2 l$

respectively. The vectors $a_{i j}$ are to be regarded as linear operators from $\lambda$ to $\mu$. Let $\mathscr{D}$ denote the diagonal submatrix of $\left(a_{i j}\right)$. Let $\mathscr{A}^{[k]}$ denote the $2 k \times 2 k$ submatrix of $\left(a_{i j}\right)$ whose $i, j$ term is $a_{i j}$ if $1 \leqq i, j \leqq 2 k$. Let $\mathscr{D}^{[k]}$ be the diagonal submatrix of $\mathscr{Q}^{[k]}$. We may regard $\mathscr{A}^{[k]}$ (and also $\mathscr{D}^{[k]}$ ) as an operator: 


$$
\mathscr{A}^{[k]}(x)=\sum_{1 \leqq j \leqq 2 k} \sum_{1 \leqq i \leqq 2 k} a_{i j}(x) \text {. }
$$

If we set $X=$ all bounded linear operators from $\lambda$ to $f^{x x}$, then we get from Lemma (2.2) that:

$$
\left\|\mathscr{C}^{[k]}\right\| \geqq\left\|\mathscr{D}^{[k]}\right\| \text {. }
$$

Here, we used the fact that $\mu^{x x}$ is a normed ideal and that $\lambda$ is monotonely normed. Assuming, momentarily, that $\mathscr{D}(\lambda) \subset \mu^{x x}$, we get that since $B=\pi_{s} \mathscr{D}$ where $S=\left\{i: m_{k} \leqq i \leqq m_{k}\right.$ for some $\left.k\right\}$ we must also have that $B(\lambda) \subset \ell^{x x}$ : it is easy to see that:

$$
\|A\| \geqq\left\|\mathscr{A}^{[k]}\right\| \geqq\left\|\mathscr{D}^{[k]}\right\|
$$

and so,

$$
\|A\| \geqq \lim _{k}\left\|\mathscr{D}^{[k]}\right\|=\|\mathscr{D}\|
$$

Thus,

$$
\|A\| \geqq\|\mathscr{D}\| \geqq\left\|\pi_{s} \mathscr{D}\right\|=\|B\|
$$

because $\mu^{x x}$ is a normed ideal.

To see that $\mathscr{D}(\lambda) \subset \mu^{x x}$, write: $x_{2 k-1}=\pi_{k}^{1}(x), x_{2 k}=\pi_{k}^{2}(x)$ for each $x \in \lambda$. Similarly, define $y_{2 k-1}^{\prime}=\pi_{k}^{1}\left(y^{\prime}\right)$ and $y_{2 k}^{\prime}=\pi_{k}^{2}\left(y^{\prime}\right)$ for each $y^{\prime} \in \mu^{x}$. For each $x \in \lambda$ and each $y^{\prime} \in \mu^{x}$, let $\kappa$ denote

$$
\left\{u \in m: u(1) x_{1} \vee \cdots \vee u(i) x_{1} \vee \cdots \in \lambda\right\} .
$$

Let $\nu^{x}$ denote

$$
\left\{v^{\prime} \in m: v^{\prime}(1) y_{1}^{\prime} \vee \cdots \vee v^{\prime}(i) y_{1}^{\prime} \vee \cdots \in \ell^{x}\right\} .
$$

Then $\kappa$ is monotonely normed and $\left(\nu^{x}\right)^{x}$ is a Banach ideal if $\kappa$ and $\nu^{x}$ are given the respective induced norms from $\lambda$ and $\mu^{x}$.

The matrix $M=\left(a_{i j}\left(x_{j}\right), y_{1}^{\prime}\right)$ defines a matrix map from $\kappa$ to 1$)^{x x}$ if we set:

$$
M(u)=\left(\sum_{1 \leqq j<\infty} u(j)\left(a_{i j}\left(x_{j}\right), y_{1}^{\prime}\right), \cdots, \sum_{1 \leqq j<\infty} u\left(j\left(\left(a_{i j}\left(x_{j}\right), y_{1}^{\prime}\right), \cdots\right)\right.\right.
$$

for each $u \in \kappa$. If $D$ is the associated diagonal of $M$, then (2.1) gives $D(\kappa) \subset \nu^{x x}$, since $\overline{1} \in \kappa$ and $\overline{1} \in \nu^{r}$, we get:

$$
\sum_{i}\left(a_{i i}\left(x_{i}\right), y_{1}^{\prime}\right)<\infty \text {. }
$$

Thus:

$$
\left(\mathscr{D}(x), y^{\prime}\right)=\sum_{i}\left(\mathscr{D}\left(x_{i}\right), y_{1}^{\prime}\right)=\sum_{i}\left(a_{i i}\left(x_{i}\right), y_{1}^{\prime}\right)<\infty
$$

holds for all $x \in \lambda, y^{\prime} \in \mu^{x}$. $\therefore \mathscr{D}(\lambda) \subset \mu^{x x}$. 
REMARKS 2.4. Although $c_{0}$ is not a perfect sequence space, it is nevertheless true that if $\lambda$ is any normal sequence space (over the reals) and $A: \lambda \rightarrow c_{0}$ is a matrix map, then the associated diagonal $D$ of $A$ maps $\lambda$ into $c_{0}$. For, if $A(\lambda) \subset c_{0}$, then Theorem (2.1) shows $D(\lambda) \subset m$. Let $\left(a_{i j}\right)$ be the matrix representation of $A$. If $r_{i}$ denotes the $i$-th row of $\left(a_{i j}\right)$, it is not hard to check that $\left\{r_{i}\right\}$ is a 0 -convergent in the weak topology on $l_{1}$ from $l_{\infty}$. Thus, $\left\{r_{i}\right\}$ is 0 -convergent in the $l_{1}$ norm. In particular,

$$
\lim \left|a_{i i}\right| \leqq \lim _{i} l_{1}\left(r_{i}\right)=0 . \quad D(\lambda) \subset c_{0} .
$$

(Essentially the same argument holds even when $\lambda \not \subset m$.)

The techniques used in (2.1) and (2.3) are similar to that of [3]. The results there were given for $\lambda=l_{p}, \mu=l_{r}$ and sharper conclusions were derived insofar as we were able to establish a criterion for $\|A\|>\|D\|$ whenever $1 \leqq r<p \leqq \infty$.

We note also that (2.3) above may be reinterpreted as showing that the projection from the space of bounded matrix maps onto the subspace of the associated diagonals is of norm one, provided that $\lambda$ is monotonely normed, while $\mu$ is a normed ideal and a perfect sequence space. Actually, we have required $\mu$ to be a normed ideal because we are proving these results in such a way as to cover sequence spaces over the complex scalars. If real scalars are being used, then it suffices to assume that $\mu$ is monotonely normed. The crucial use of these hypotheses is in facilitating the use of Lemma (1.3) where the definition of $y^{\prime}$ following (1.3.1) is the deciding issue.

3. This section gives an independent proof of Theorem (2.1) for the case where $\lambda$ is normal. With this hypothesis on $\lambda$, we are able to reduce the problem to an argument involving matrix maps between Banach sequence spaces and thereby avoid the involved computations of (2.1). We allow $\lambda$ and $\mu$ to be sequence spaces over the reals or the complex numbers. The rest of the section gives the application to the problem of Mazur.

THeorem 3.1. Let $\lambda$ be a normal sequence space. Let $\mu$ be any sequence spaces. Let $A: \lambda \rightarrow \mu$ be a matrix map. Then the associated diagonal matrix $D$ of $A$ maps $\lambda$ into $\mu^{x x}$.

Proof. If $u \in \lambda$ and $v^{\prime} \in \mu^{x}$, define $\kappa, \nu$ to be Banach sequence spaces by setting:

$$
\begin{aligned}
\kappa & =\{x \in \omega: x \cdot u \in m\} \\
\|x\|_{\kappa} & =\sup \left\{\frac{|x(k)|}{|u(k)|}: k=1,2, \cdots\right\}
\end{aligned}
$$




$$
\begin{aligned}
\nu & =\left\{y \in \omega: \sum_{k}\left|y(k) v^{\prime}(k)\right|<\infty\right\} \\
\|y\|_{\nu} & =\sum_{k}\left|y(k) v^{\prime}(k)\right| .
\end{aligned}
$$

Here, we have assumed without loss of generality, that $u(k) \neq 0$, $v^{\prime}(k) \neq 0$ for all $k$. It is easy to check that $\kappa$ and $\nu$ are Banach ideals. Clearly, $A(\kappa) \subset \nu$ and since any matrix map between Banach sequence spaces is bounded (see Corollary 5, p. 204 of [4]), $A$ is also a bounded operator.

The theorem is proved if we show that $D(\kappa) \subset \nu$. Let $\|\cdot\|$ denote the operator sup norm. If $D$ does not map $\kappa$ into $\nu$, then:

$$
\sum_{k}\left|a_{k k} u(k) v^{\prime}(k)\right|=\infty \text {. }
$$

By (1.5), we have:

$$
\begin{aligned}
\infty>\|A\| & \geqq \sup _{n}\left\|\pi_{[1, n]} A \pi_{[1, n]}\right\| \\
& \geqq \sup _{n}\left\|\pi_{[1, n]} D \pi_{[1, n]}\right\| \geqq\|D\| \\
& \geqq \sum_{k}\left|a_{k k} u(k) v^{\prime}(k)\right|=\infty .
\end{aligned}
$$

Hence, we must have had: $D(\kappa) \subset \nu$.

If $\mu$ is a normed sequence space we give $\mu^{x x}$ the norm generated by (unit ball of $\mu)^{x x}$.

THEOREM 3.2. Let $\lambda$ be a monotonely normed sequence space. Let $\mu$ be any sequence space. Let $A: \lambda \rightarrow \mu$ be a bounded matrix map. If $D$ is the associated diagonal of $A$, then $D(\lambda) \subset \mu^{x x}$ and $\|A\| \geqq\|D\|$, where the sup norm is computed with respect to the norms of $\lambda$ and $\mu^{x x}$.

Proof. By (2.1), $D(\lambda) \subset u^{x x}$. Observe that

$$
\|D\|=\lim _{n}\left\|\pi_{[1, n]} D \pi_{[1, n]}\right\|
$$

and argue as in (3.1.1), observing that $\pi_{[1, n]}(\lambda)$ is a monotonely normed sequence (of dimension $n$ ) and that $\pi_{[1, n]}(\mu)$ is a normed ideal (of dimension $n$ ) so that the appeal to (1.5) is legitimate.

S. Mazur posed and affirmatively answered the following problem: If $\left(a_{i j}\right)$ is a matrix of complex numbers so that $\sum_{1}\left|\sum_{j} a_{i j} x(j)\right|<\infty$ for all $x \in m$, does it follow that $\sum_{1}\left|a_{i i}\right|<\infty$ ?

His answer, found in Pelczyński-Szlenk [2], gives a stronger conclusion. In this section, we apply our results in $\S 2$ to give a more general version of the problem. 
THEOREM 3.3. Let $1 \leqq p, r \leqq \omega$. Let $g$ denote $\infty, p r /(p-r)$, or $r$ depending on whether $1 \leqq p \leqq r \leqq \infty, 1 \leqq r<p<\omega$, or $1 \leqq$ $r<\infty$ and $p=\infty$ respectively. Let $A: l_{p} \rightarrow l_{r}$ be a matrix map. Then $l_{g}\left(a_{11}, \cdots a_{i i}, \cdots\right)<\infty$. In fact, if $D$ is the associated diagonal of $A$, then $D\left(l_{p}\right) \subset l_{r}$ and $\|A\| \geqq\|D\|$.

Proof. Note that for the case $p=\infty, r=1$, we have $g=1$ so that this theorem contains the affirmative answer of Mazur. Observing that $l_{r}=l_{r}^{x x}$, we conclude from (2.1) that $D\left(l_{p}\right) \subset l_{r}$. In (2.2) of [3], it was computed that $\|D\|=l_{y}\left(a_{11}, \cdots, a_{i i}, \cdots\right)$ and an inspection of the proof shows that $D\left(l_{p}\right) \subset l_{r}$ if and only if $\|D\|<\omega$. Finally,

$$
\begin{aligned}
\|A\| & \geqq \lim _{n}\left\|\pi_{\lfloor 1, n]} A \pi_{[1, n]}\right\| \\
& \geqq \lim _{n}\left\|\pi_{[1, n]} D \pi_{[1, n]}\right\| \\
& =\lim _{n} l_{g}\left(a_{11}, \cdots, a_{n n}\right)=\|D\|
\end{aligned}
$$

where (3.2) was used to obtain the second inequality. We note in passing that a Baire category argument proves every matrix map (between Banach sequence spaces) to be bounded (see Corollary 5, p. 204 of [4]) so that in the theorem above, $\|D\| \leqq\|A\|<\infty$.

\section{BIBLIOGRAPHY}

1. G. Kothe, T'opologische lineare Räume, Springer, 1960.

2. A. Pelczyński and W. Szlenk, Sur l'injection de espaces $(l)$ dans l'espace $\left(l_{p}\right)$, Colloquium Mathematicum, 10 (1963), 313-323.

3. A. Tong, Diagonal nuclear operators on $l_{p}$ spaces (to appear in Trans. Amer. Math. Soc.)

4. A. Wilansky, Functional analysis, Blaisdell, 1964.

Received February 18, 1969.

State University of New York at Albany 



\section{PACIFIC JOURNAL OF MATHEMATICS}

\section{EDITORS}

\author{
H. SAMELSON \\ Stanford University \\ Stanford, California 94305 \\ RICHARD PIERCE \\ University of Washington \\ Seattle, Washington 98105
}

J. DugundJI

Department of Mathematics University of Southern California Los Angeles, California 90007

BASIL GORDON*

University of California

Los Angeles, California 90024

\section{ASSOCIATE EDITORS}
E. F. BECKENBACH
B. H. NEUMANN
F. WOLE
K. YOSHIDA

\section{SUPPORTING INSTITUTIONS}

\author{
UNIVERSITY OF BRITISH COLUMBIA \\ CALIFORNIA INSTITUTE OF TECHNOLOGY \\ UNIVERSITY OF CALIFORNIA \\ MONTANA STATE UNIVERSITY \\ UNIVERSITY OF NEVADA \\ NEW MEXICO STATE UNIVERSITY \\ OREGON STATE UNIVERSITY \\ UNIVERSITY OF OREGON \\ OSAKA UNIVERSITY \\ UNIVERSITY OF SOUTHERN CALIFORNIA
}

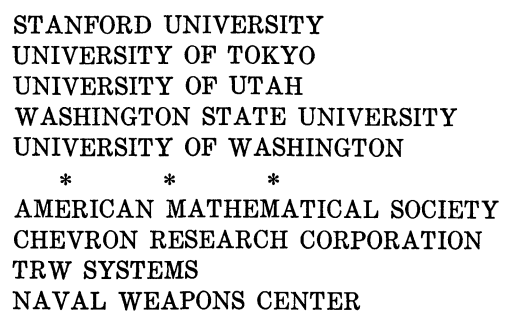

The Supporting Institutions listed above contribute to the cost of publication of this Journal, but they are not owners or publishers and have no responsibility for its content or policies.

Mathematical papers intended for publication in the Pacific Journal of Mathematics should be in typed form or offset-reproduced, (not dittoed), double spaced with large margins. Underline Greek letters in red, German in green, and script in blue. The first paragraph or two must be capable of being used separately as a synopsis of the entire paper. The editorial "we" must not be used in the synopsis, and items of the bibliography should not be cited there unless absolutely necessary, in which case they must be identified by author and Journal, rather than by item number. Manuscripts, in duplicate if possible, may be sent to any one of the four editors. Please classify according to the scheme of Math. Rev. 36, 1539-1546. All other communications to the editors should be addressed to the managing editor, Richard Arens, University of California, Los Angeles, California, 90024.

50 reprints are provided free for each article; additional copies may be obtained at cost in multiples of 50 .

The Pacific Journal of Mathematics is published monthly. Effective with Volume 16 the price per volume (3 numbers) is $\$ 8.00$; single issues, $\$ 3.00$. Special price for current issues to individual faculty members of supporting institutions and to individual members of the American Mathematical Society: $\$ 4.00$ per volume; single issues $\$ 1.50$. Back numbers are available.

Subscriptions, orders for back numbers, and changes of address should be sent to Pacific Journal of Mathematics, 103 Highland Boulevard, Berkeley, California, 94708.

PUBLISHED BY PACIFIC JOURNAL OF MATHEMATICS, A NON-PROFIT CORPORATION

Printed at Kokusai Bunken Insatsusha (International Academic Printing Co., Ltd.), 7-17, Fujimi 2-chome, Chiyoda-ku, Tokyo, Japan.

* Acting Managing Editor. 


\section{Pacific Journal of Mathematics}

\section{Vol. 32, No. $2 \quad$ February, 1970}

Harry P. Allen and Joseph Cooley Ferrar, Jordan algebras and exceptional subalgebras of the exceptional algebra $E_{6} \ldots \ldots \ldots \ldots \ldots \ldots \ldots 283$

David Wilmot Barnette and Branko Grünbaum, Preassigning the shape of a

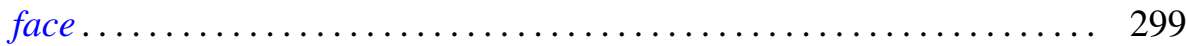

Robert Francis Craggs, Involutions of the 3-sphere which fix 2-spheres . . . . 307

David William Dean, Bor-Luh Lin and Ivan Singer, On k-shrinking and $k$-boundedly complete bases in Banach spaces ................ 323

Martin Engert, Finite dimensional translation invariant subspaces ....... 333

Kenneth Lewis Fields, On the global dimension of residue rings ......... 345

Howard Gorman, The Brandt condition and invertibility of modules ....... 351

Benjamin Rigler Halpern, A characterization of the circle and interval ..... 373

Albert Emerson Hurd, A uniqueness theorem for second order quasilinear

hyperbolic equations ............................... 415

James Frederick Hurley, Composition series in Chevalley algebras ...... 429

Meira Lavie, Disconjugacy of linear differential equations in the complex

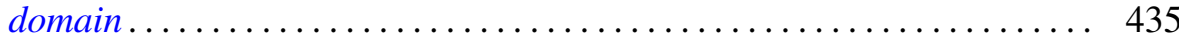

Jimmie Don Lawson, Lattices with no interval homomorphisms ......... 459

Roger McCann, A classification of center-foci ................. 467

Evelyn Rupard McMillan, On continuity conditions for functions . . . . . . . 479

Graciano de Oliveira, A conjecture and some problems on permanents .... 495

David L. Parrott and S. K. Wong, On the Higman-Sims simple group of order $44,352,000$.

Jerome L. Paul, Extending homeomorphisms ................. 517

Thomas Benny Rushing, Unknotting unions of cells .............. 521

Peter Russell, Forms of the affine line and its additive group.......... 527

Niel Shilkret, Non-Archimedean Gelfand theory ................. 541

Alfred Esperanza Tong, Diagonal submatrices of matrix maps.......... 551 